\title{
Bilevel programming approaches to production planning for multiple products with short life-cycles
}

\author{
Xide Zhu • Peijun Guo
}

Received: date / Accepted: date

\begin{abstract}
We provide decision-making models for a manufacturer which plans to produce multiple short life cycle products with the one-shot decision theory. The obtained optimal production quantities are based on the most appropriate scenarios for the manufacturer. Since the models are the bilevel programming problems with the max-min or min-max operator in the lower levels, we propose two approaches to translate them into general single-level optimization problems such that they can be solved via the commonly used optimization methods. The effectiveness of our approaches is examined from the theoretical and computational aspects.
\end{abstract}

Keywords Bilevel programming · One-shot decision theory · Production planning · Uncertainty

Mathematics Subject Classification (2000) 90B50 - 90C26 - 90C30 • 91B06

\section{Introduction}

The production planning problem is a fundamental and important managerial decision-making problem in various industries such as agricultural industry, manufacturing industry, entertainment industry, etc. Since production planning problems in the real world invariably include some unknown parameters, uncertainty is a main factor that affects the effectiveness of the obtained

Xide Zhu

Faculty of Business Administration, Yokohama National University, 79-4 Tokiwadai, Hodogaya-ku, Yokohama 240-8501, Japan.

E-mail: xidezhu@gmail.com

Peijun Guo, Corresponding author

Faculty of Business Administration, Yokohama National University, 79-4 Tokiwadai, Hodogaya-ku, Yokohama 240-8501, Japan.

Tel.: +81 453393677

E-mail: guo@ynu.ac.jp 
plan. Uncertainty involved in production planning problems can generally be categorized into two major types: system uncertainty and environmental uncertainty. The former includes uncertainties within the production processes, such as quality uncertainty, operation yield uncertainty and so on; the latter involves uncertainties beyond the production processes, such as demand uncertainty, supply uncertainty and so on [37]. The uncertainty in production planning problems was first examined by Dantzig [20]. From then on, a considerable amount of research (e.g., $[5,27,34,36,40,55,56,60])$ and surveys have appeared in the production planning literature, including those of Mula et al. [45], and Wazed, Ahmed and Nukman [64].

From the aspect of mathematical optimization, production planning problems involving uncertainty can be mainly modeled by the following approaches. The first approach is stochastic programming where the uncertain parameters can be characterized by random variables whose probability distributions are known or can be estimated $[13,41,46]$. Specifically, there are two kinds of methods to deal with stochastic programming problems: chance-constrained methods and recourse methods. The chance-constrained methods ensure that the optimal solution makes the probability of a certain constraint being satisfied above a certain level $[16,44,47,50]$. The recourse methods are mainly used in two-stage (or multi-stage) problems: in the first stage, a feasible solution is chosen before observing the random parameters; in the second stage, upon a realization of the random parameters, further decisions are allowed to avoid the infeasibility of constraints $[11,54]$. The second approach is robust optimization to deal with the uncertain parameters which are known to reside in (bounded) uncertainty sets $[1,4-6,15]$. According to different decision environments, this kind of problem can be subdivided as static robust optimization or adjustable robust optimization. Static robust optimization is for the case that all decision variables represent here-and-now decisions, that is, they should be made before the actual parameters are observed. Adjustable robust optimization is for the case that the part of the decision variables must be determined before the realization of the uncertain parameters, while the others can be adjusted after some parts of the uncertain parameters are revealed $[9,26]$. The paper [10] gives a comprehensive overview of robust optimization theory and applications. In addition, distributionally robust optimization approach is also widely used for the case that the uncertain parameters can be characterized by random variables but the probability distributions are not fully known due to the lack of enough historical data. Distributionally robust optimization provides an alternative way to overcome the conservativeness of the robust optimization without requiring exact specifications of the probability distributions [35, $65,68]$.

Uncertain optimization problems, especially multi-stage models, often raise computational complexity. In order to overcome these computational difficulties, a new approximation approach is proposed via linear or nonlinear decision rules. Since the approximate models are computationally tractable and retain the fundamental structure of the problem, decision rule approaches have gradually drawn the attention of researchers in the last decade or so; see, e.g., [9, 
$17,18]$. In a recent paper [66], the authors applied this decision rule approach to a two-stage stochastic bilevel programming problem and proposed two approximation problems that are easier to be solved than the original problem.

In this paper, we consider a production planning problem for innovative products as defined by Fisher [24]. According to Fisher, an innovative product has a higher profit margin, an intrinsically unpredictable demand and a short life cycle. In addition, for such an innovative product, the procurement leadtime is usually longer than the selling season so that there is often only one opportunity to produce goods before the season. One typical example is fashion clothes which are characterized by volatile and unpredictable demands, short life cycles and long supply processes [53]. Fashion items are sold punctually in a short period and generally not replenished so that they are called as oneshot items [61]. Hence, the production planning problem for such products is typically a one-shot decision problem.

We build production planning models with the one-shot decision theory which is proposed by Guo [31]. The one-shot decision theory has been utilized for analyzing a duopoly market of a new product with a short life cycle $[29,30]$, newsvendor problems for innovative products [32], multi-stage one-shot decision making problems [33] and first-price sealed-bid auctions [63]. For the sake of simplicity, we only consider the market uncertainty which is characterized by a random vector of unit profits of innovative products and all decision variables (the production levels of all products) represent here-and-now decisions. The one-shot decision theory based production planning models (ODPPMs) obtain the optimal solution by a two-step process shown below. First, for each feasible production level, the manufacturer examines every realization of random vector (unit profits of products) with considering the probability of this realization and the outcome associated with it and chooses one as a focus point of this production level. The selected focus point is the most appropriate scenario for the manufacturer executing this production level. Second, the manufacturer determines such a production level as the optimal one that generates the highest total profit when its focus point occurs. This focus point is a supporting scenario for the optimal production level determined. In other words, the reason that a production level is chosen as an optimal one is that its focus point is regarded as the most appropriate scenario for the manufacturer.

ODPPMs are bilevel programming problems (BLPPs) where the upper level problem is to determine the optimal production level of each product and the lower level problem is to choose the focus point of each production level. BLPP is an important optimization problem, which plays an important role in many fields such as transportation, economics, business, engineering, decision science and so on [21]. It has been known that it is generally difficult to solve BLPPs because their constraints include a series of other optimization problems $[8,19]$. The commonly used approach to a BLPP is to translate it into a mathematical program with equilibrium constraints (MPEC) based on the KKT reformulation. Since it is difficult to solve ODPPMs by the KKT based method, we propose two new approaches by which we can translate the 
ODPPMs into general single-level optimization problems so that they can be solved via the commonly used optimization methods and software.

Briefly, the contribution of this research is summarized as follows. (1) From the operational research aspect, a new production planning model for multiple products with short life-cycles is proposed to reflect the one-shot feature of producing these products, it is fundamentally different from the existing production planning models. The proposed production planning models are BLPPs in which the lower level problem is for finding out the most appropriate prices of all products for each production level, while considering its probability and the profit caused by it, which is called the focus point of this production level; the upper level problem is to decide the optimal production level based on all focus points of all production levels. (2) From the computational aspect, new solution methods are proposed to solve the ODPPMs with the max-min or min-max operator in the lower levels. The proposed singlelevel equivalence models are more tractable than traditional KKT condition based reformulations. Hence, they can be efficiently solved by the classic numerical methods or software. To the best of our knowledge, there is no such kind of BLPPs which has been solved.

The remainder of this paper is organized as follows. In Section 2, we model production planning problems under uncertainty with the one-shot decision theory and compare the proposed approaches with several existing methods including robust optimization, the expected utility based method. In Section 3 , two methods are proposed for effectively solving the models. In Section 4, a numerical example is used to show the effectiveness of the proposed methods, a comparison with other methods is made, and the managerial insights are gained. Finally, we conclude our research in Section 5.

\section{Production planning models with the one-shot decision theory}

Consider a manufacturer who is making a production planning for multiple innovative products with short life-cycles under market uncertainty. The production quantity of product $i(i=1,2, \cdots, n)$ is the decision variable $x_{i}$, the unit profit of product $i$ is a random variable $\Xi_{i}$ and its realization (a scenario) is $\xi_{i}$. Assuming that the feasible region of $\xi$ is $\Omega:=\left[\xi_{l}, \xi_{u}\right] \subset R^{n}$ with $\xi_{l}<\xi_{u}$ where $\xi_{l}$ and $\xi_{u}$ are the lower and upper bounds of $\xi$, respectively. $X:=\{x: A x \leq b, x \geq 0\} \subset R^{n}$ represents the constraints of available resources, such as time, materials, etc.

2.1 The existing models for production planning problems

Such a production planning problem is often modeled as the following stochastic optimization problem:

$$
\max _{x} \Xi^{T} x \quad \text { s.t. } x \in X .
$$


Since $\Xi$ is a random vector, the meaning of 'max' is not clear at all. That is, the problem (1) is not well defined. Basically, it will be reformulated as deterministic optimization models based on the different consideration about $\Xi^{T} x$. In the following, we briefly overview existing optimization models for it.

The expected profit based method indicates that the optimal choice of $x$ is the solution of the following optimization problem:

$$
\max _{x} \overline{\Xi^{T} x} \quad \text { s.t. } x \in X,
$$

where $\overline{\Xi^{T} x}$ represents the expectation of $\Xi^{T} x$. Since $\overline{\Xi^{T} x}=\bar{\Xi}^{T} x$ for any $x \in X$, we can rewrite (2) as a deterministic linear programming problem:

$$
\max _{x} \bar{\Xi}^{T} x \quad \text { s.t. } x \in X .
$$

Now let us consider a simple example as follows:

$$
\max _{x} \Xi_{1} x_{1}+\Xi_{2} x_{2} \quad \text { s.t. } 3 x_{1}+x_{2} \leq 9, x_{1}+2 x_{2} \leq 8, x_{1}, x_{2} \in N .
$$

Suppose that $\Xi_{1}$ and $\Xi_{2}$ are independent, discrete random variables and the probability distributions of $\Xi_{1}$ and $\Xi_{2}$ are given as $P\left(\Xi_{1}=1\right)=0.6, P\left(\Xi_{1}=\right.$ $13)=0.4$ and $P\left(\Xi_{2}=3\right)=0.3, P\left(\Xi_{2}=4\right)=0.7$, respectively. We can obtain that the joint probability distribution of the random vector $\Xi:=\left(\Xi_{1} ; \Xi_{2}\right)$ as $P(\Xi=(1 ; 3))=0.18, P(\Xi=(1 ; 4))=0.42, P(\Xi=(13 ; 3))=0.12$, $P(\Xi=(13 ; 4))=0.28$, and the expectation of $\Xi$ is $\Xi=(5.8 ; 3.7)$. Further, we can obtain the optimal solutions corresponding to four scenarios of $(4)$, that is, $x^{*}((1 ; 3))=(0 ; 4), x^{*}((1 ; 4))=(0 ; 4), x^{*}((13 ; 3))=(3 ; 0)$ and $x^{*}((13 ; 4))=$ $(3 ; 0)$ while the global optimal solution of $(2)$ is $x^{*}(\bar{\Xi})=(2 ; 3)$. Although the optimal solution of (2) may not correspond to any possible realization in the future, by the Law of Large Numbers, if the process repeats over a large number of times, then the solution of (2) will be optimal on average. Indeed, in this case, it makes sense to consider using the expected value method to solve a stochastic optimization problem [54].

Another method for solving (1) is called the max-max optimization approach where the optimal choice of $x$ is the solution of the following max-max optimization problem:

$$
\max _{x} \max _{\xi}\left\{\xi^{T} x: \xi \in \Omega\right\} \quad \text { s.t. } x \in X .
$$

The max-max approach is an important decision criterion assuming that the best scenario will happen whatever action is taken. This approach is appropriate for an optimistic decision-maker who is often attracted by the best results. For any $x \geq 0$, we have $\xi_{u}^{T} x=\max \left\{\xi^{T} x: \xi \in \Omega\right\}$, which implies that (5) is equivalent to the following deterministic linear programming problem:

$$
\max _{x} \xi_{u}^{T} x \quad \text { s.t. } x \in X .
$$


Considering the example $(4)$, we have $\xi_{u}=(13 ; 4)$ and $x^{*}((13 ; 4))=(3 ; 0)$. That is, the best scenario is $(13 ; 4)$, and $(3 ; 0)$ is obtained as an optimal solution to correspond to this scenario.

The other method for solving (1) is called the max-min optimization approach where the optimal choice of $x$ is the solution of the following max-min optimization problem:

$$
\max _{x} \min _{\xi}\left\{\xi^{T} x: \xi \in \Omega\right\} \quad \text { s.t. } x \in X
$$

The max-min approach is another important decision criterion which assumes that the worst scenario will appear whatever action is taken. The max-min approach would be suitable for a pessimistic decision-maker who is always worried about the worst results. Similarly, for any $x \geq 0$, we have $\xi_{l}^{T} x=$ $\min \left\{\xi^{T} x: \xi \in \Omega\right\}$, hence $(7)$ can be written as the following deterministic linear programming problem:

$$
\max _{x} \xi_{l}^{T} x \quad \text { s.t. } x \in X .
$$

Considering the example $(4)$, we have $\xi_{l}=(1 ; 3)$ and $x^{*}((1 ; 3))=(0 ; 4)$. That is, $(1 ; 3)$ is the worst scenario and $(0 ; 4)$ is obtained as an optimal solution to correspond to it.

Clearly, the max-max optimization approach might be too daring whereas the max-min optimization approach might be too conservative in the sense that the former only considers the best scenario but the latter only takes into account the worst one no matter what the probabilities of them are. We call the manufacturer who adopts the optimal production level formulated by (2), (5) or (7) as the expected profit maximization manufacturer, the max-max manufacturer or the max-min manufacturer, respectively.

\subsection{The one-shot decision theory based models}

We model the production planning problem for innovative products with short life-cycles where the profits per unit of products are uncertain. Due to the short life cycles of these products, one and only one realization of a random vector of the unit profits of these products (a scenario) will appear in the future and the manufacturer has only one opportunity to determine the production levels before the scenario reveals. Considering the one-time feature of such problems, we build the ODPPM to obtain the optimal production level by the following two steps. First, for each feasible production level given by the upper level problem, the lower level problem examines every possible realization of the random vector of unit profits of products with considering the probability of this realization and the outcome associated with it and chooses one as a focus point of this production level (an optimal solution of the lower level problem). Then, the upper level problem determines such a production level as the optimal one that generates the highest total profit with its focus point. 
We consider two types of behaviors of the manufacturer choosing focus points: one is choosing the scenario (one realization of the random vector) which has a relatively high probability and can bring about a relatively high profit as the active focus; the other is choosing the scenario which has a relatively high probability but can lead to a relatively low profit as the passive focus. We formulate these two types of focus points as follows.

- The active focus point of $x$, denoted as $\xi^{a c}(x)$, is a non-dominated optimal solution of the following two-objective optimization problem:

$$
\left(\max _{\xi} f(\xi) ; \max _{\xi} \xi^{T} x\right) \quad \text { s.t. } \xi \in \Omega,
$$

where $f(\xi)$ is the objective probability mass (density) function for discrete (continuous) random vector or the subjective probability exogenously given by the manufacturer representing the degree of belief on the occurrence of each scenario, and $\xi^{T} x$ is the payoff function.

- The passive focus point of $x$, denoted as $\xi^{p a}(x)$, is a non-dominated optimal solution of the following two-objective optimization problem:

$$
\left(\max _{\xi} f(\xi) ; \min _{\xi} \xi^{T} x\right) \quad \text { s.t. } \xi \in \Omega
$$

(9) and (10) reflect the optimistic and pessimistic attitudes to evaluate the scenario, respectively. We call the manufacturer who takes into account the active focus point or passive focus point as active manufacturer or passive manufacturer. The manufacturer considers the focus point as his/her most appropriate scenario for each feasible production level and then chooses the optimal production level that generates the highest total profit when its focus point occurs. Based on the above considerations, we formulate the production planning problems for these two kinds of manufacturers as follows.

- The production planning model with active focus points:

$$
\max _{x} \xi^{a c}(x)^{T} x \quad \text { s.t. } x \in X .
$$

- The production planning model with passive focus points:

$$
\max _{x} \xi^{p a}(x)^{T} x \quad \text { s.t. } x \in X .
$$

The solutions of (11) and (12) are called the active optimal production level and the passive optimal production level, respectively. It should be noted that we only consider one non-dominated solution of (9) or (10) for each $x \in X$ in (11) or (12). In other words, we do not take into account the frontier of (9) or (10). We have the following lemma to clarify the relationship between the production planning model with active focus points and the max-max optimization model and the relationship between the production planning model with passive focus points and the max-min optimization model.

Lemma 1 The max-max optimization approach (5) is the special case of (11); the max-min optimization approach (7) is the special case of (12). 
Proof It is easy to verify that $\xi_{u}$ is Pareto optimal for the two-objective optimization problem (9) since $\xi_{u}^{T} x=\max \left\{\xi^{T} x: \xi \in \Omega\right\}$ for any $x \geq 0$. In other words, if the best scenario $\xi_{u}$ is chosen by the manufacturer as the active focus point of all feasible $x$, i.e., $\xi^{a c}(x) \equiv \xi_{u}$, then the production planning model with active focus points reduces to the linear programming problem (6). Similarly, we can verify that $\xi_{l}$ is Pareto optimal for the two-objective optimization problem (10) since $\xi_{l}^{T} x=\min \left\{\xi^{T} x: \xi \in \Omega\right\}$ for all feasible $x$. Hence the production planning model with passive focus points can be reduced to the linear programming problem (8) if $\xi^{p a}(x) \equiv \xi_{l}$.

It follows from Lemma 1 that max-max and max-min optimization approaches completely ignore probabilities. In what follows, we show other approaches which can obtain a non-dominated optimal solution of (9) and (10) with considering profits and probabilities simultaneously. First, we give two definitions as follows.

Definition 1 Let $f$ be the original probability mass (density) function for discrete (continuous) random vector and $\pi: \Omega \rightarrow[0,1]$ be a function satisfying

$$
\max \{\pi(\xi): \xi \in \Omega\}=1 .
$$

We call $\pi(\xi)$ as the relative likelihood degree of $\xi$ if it satisfies $\pi\left(\xi_{1}\right)>\pi\left(\xi_{2}\right)$ for $f\left(\xi_{1}\right)>f\left(\xi_{2}\right)$ and $\pi\left(\xi_{1}\right)=\pi\left(\xi_{2}\right)$ for $f\left(\xi_{1}\right)=f\left(\xi_{2}\right)$.

Definition 2 Let $v$ be the payoff function and $u: \Omega \times X \rightarrow[0,1]$ be a function satisfying

$$
\max \{u(\xi, x):(\xi, x) \in \Omega \times X\}=1 .
$$

We call $u(\xi, x)$ as the satisfaction level of $\xi$ for $x$ if it satisfies $u\left(\xi_{1}, x\right)>u\left(\xi_{2}, x\right)$ for $v\left(\xi_{1}, x\right)>v\left(\xi_{2}, x\right)$ and $u\left(\xi_{1}, x\right)=u\left(\xi_{2}, x\right)$ for $v\left(\xi_{1}, x\right)=v\left(\xi_{2}, x\right)$.

Clearly, the relative likelihood degree and the satisfaction level are used to represent the relative position of probability and payoff, respectively.

In fact, there are many kinds of probability distributions. Considering the fact that most commonly used probability distributions have a log-concave density function [7] (e.g., normal distributions, exponential distributions), we assume hereafter that $\Xi$ follows a (truncated) log-concave distribution with a given support $\Omega=\left[\xi_{l}, \xi_{u}\right]$. Based on Definitions 1 and 2, we can give two formulas as follows:

$$
\begin{gathered}
\pi(\xi):=\frac{1}{\log \left(f_{u}\right)-\log \left(f_{l}\right)}\left(\log (f(\xi))-\log \left(f_{l}\right)\right), \\
u(\xi, x):=\frac{1}{v_{u}-v_{l}}\left(\xi^{T} x-v_{l}\right),
\end{gathered}
$$

where $f_{l}$ and $f_{u}$ are lower and upper bounds of $f(\xi)$ in $\Omega$ respectively, i.e.,

$$
f_{l}:=\min \{f(\xi): \xi \in \Omega\} \text { and } f_{u}:=\max \{f(\xi): \xi \in \Omega\} ;
$$


$v_{l}$ and $v_{u}$ are lower and upper bounds of $\xi^{T} x$ in $\Omega \times X$ respectively, i.e.,

$$
v_{l}:=\min \left\{\xi^{T} x: x \in X, \xi \in \Omega\right\} \text { and } v_{u}:=\max \left\{\xi^{T} x: x \in X, \xi \in \Omega\right\} .
$$

Instead of directly normalizing the original density function, we utilize (13) as the relative likelihood function because it is a concave function and hence it is computationally tractable.

With $\pi(\xi)$ and $u(\xi, x)$, we give another specific non-dominated solution of (9) as follows:

$$
\max _{\xi \in \Omega} \min \{\pi(\xi), u(\xi, x)\}
$$

From (13) and (14), we can easily verify that the solutions of (15) are still Pareto optimal for (9) for any $x \in X$. Since the minimal function between $\pi$ and $u$ can be expressed as

$$
\min \{\pi(\xi), u(\xi, x)\}=\frac{1}{2}\{\pi(\xi)+u(\xi, x)-|\pi(\xi)-u(\xi, x)|\},
$$

it is straightforward that using (15) we can find out a scenario $\xi$ which simultaneously makes $\pi(\xi)$ and $u(\xi, x)$ large. In other words, comparing with $\max _{\xi \in \Omega}\{\pi(\xi)+u(\xi, x)\}$ and $\max _{\xi \in \Omega}\{\pi(\xi) * u(\xi, x)\}$, (15) can avoid obtaining $\xi$ with a large $\pi(\xi)$ but a small $u(\xi, x)$ or a small $\pi(\xi)$ but a large $u(\xi, x)$ because it tries to make $|\pi(\xi)-u(\xi, x)|$ as smaller as possible. Similarly, by solving

$$
\min _{\xi \in \Omega} \max \{1-\pi(\xi), u(\xi, x)\}
$$

we can obtain another specific non-dominated solution of (10). Considering

$$
\max \{1-\pi(\xi), u(\xi, x)\}=\frac{1}{2}\{1-\pi(\xi)+u(\xi, x)+|1-\pi(\xi)-u(\xi, x)|\},
$$

we know that using (16) we can find out a scenario $\xi$ with a relatively large $\pi(\xi)$ and a relatively small $u(\xi, x)$.

With (15) and (16), we respectively embody the ODPPM with active focus points and the ODPPM with passive focus points by Model A and Model B as shown below.

\section{Model A:}

$$
\max _{(x ; \xi)} \xi^{T} x \quad \text { s.t. } x \in X, \xi \in \Lambda^{a c}(x),
$$

where $\Lambda^{a c}(x)$ (which is related to $x$ ) denotes the set of global optimal solutions of the following max-min lower level problem:

$$
\max _{\xi \in \Omega} \min \{\pi(\xi), u(\xi, x)\} .
$$

Clearly, for Model A, the upper level problem (17) is used to find the optimal production level for maximizing the total profit on a specific scenario associated with it; the lower level problem (18) is used to seek this scenario which 
has a relatively high probability and can cause a relatively high profit. Considering the example (4), we can obtain that the active optimal production level as $x^{*}=(3 ; 0)$ and its focus point as $\xi^{a c}\left(x^{*}\right)=(13 ; 4)$. It means that the active manufacturer chooses the production level $(3 ; 0)$ as the optimal one based on one scenario $(13 ; 4)$ (profits per units of two products). The relative likelihood degree of $(13 ; 4)$ is 0.6763 and it can lead to the satisfaction level of 1.0000 if the production level is $(3 ; 0)$.

Model B:

$$
\max _{(x ; \xi)} \xi^{T} x \quad \text { s.t. } x \in X, \xi \in \Lambda^{p a}(x)
$$

where $\Lambda^{p a}(x)$ (which is related to $x$ ) denotes the set of global optimal solutions of the following min-max lower level problem:

$$
\min _{\xi \in \Omega} \max \{1-\pi(\xi), u(\xi, x)\} .
$$

Likewise, for Model B, the upper level problem (19) is used to find the optimal production level for maximizing the total profit on a specific scenario associated with it; the lower level problem (20) is used to seek this scenario which has a relatively high probability and can cause a relatively low profit. Considering the example (4), we can obtain that the passive optimal production level as $x^{*}=(0 ; 4)$ and its focus point as $\xi^{p a}\left(x^{*}\right)=(1 ; 4)$. It means that the passive manufacturer chooses the production level $(0 ; 4)$ as the optimal one based on one scenario $(1 ; 4)$ (profits per units of two products). The relative likelihood degree of $(1 ; 4)$ is 1.0000 and it can lead to the satisfaction level of 0.4102 if the production level is $(1 ; 4)$.

Remarks: Comparing with the max-max optimization approach and the maxmin optimization approach, although Model A and Model B utilize the operator 'max-min' and 'min-max', they also incorporate the probability, and so they simply eliminate the possibility of obtaining extreme results, that is, too optimistic results from the max-max optimization approach or too conservative results from the max-min optimization approach. The expected utility based method is for the decision maker whose action can be characterized by four axioms (completeness, transitivity, independence and continuity) of von Neumann and Morgenstern [62] or nine axioms (completeness, reflexivity, transitivity, sure thing principle, monotonicity, weak comparative, nondegeneracy, small event continuity and uniform monotonicity) of Savage [51]. However, plenty of evidence shows that people systematically violate these axioms (see, e.g. Allais [2]; Ellsberg [25]; Kahneman and Tversky [39]; Starmer [57]). ODPPMs are behavioral models with two basic assumptions (axioms): for each action, there is an event which is personally most salient for the decision maker; the optimal action is determined by comparing the salient events of all actions (Guo [31]). Actually, there are mounting evidence to support these assumptions (for example, Orquin and Loose [48]; Stewart, Hermens and Matthews [59]; Busse et al. [14]; Bordalo, Gennaioli and Shleifer [12]); in addition, a behavioral model with the one-shot decision theory is built for 
explaining the anomalies in the first-price sealed-bid auctions, especially, it is the first theoretical explanation for throwing-away phenomenon (Wang and Guo [63]). In summary, with different assumptions, we can utilize different production planning models, such as the max-max optimization model, the max-min optimization model, the expected utility based model and the oneshot decision theory based model; ODPPM provides an alternative strategy for the manufacturer choosing the optimal production level.

\section{The solutions to Models A and B.}

From Model A and Model B, we know that solving these models is to solve the BLPPs with non-smooth lower level problems. To overcome the difficulty of non-smoothing, we first introduce a new auxiliary variable $y$ where $y \leq$ $\min \{\pi(\xi), u(\xi, x)\}$. Let us consider the following optimization problem:

$$
\max _{(\xi ; y)} y \quad \text { s.t. } y \leq \pi(\xi), y \leq u(\xi, x),(\xi ; y) \in\left[\xi_{l}, \xi_{u}\right] \times R
$$

Lemma 2 Let $\Omega(x) \subset R^{n} \times R$ be the set of global optimal solutions of (21) for any $x \in X$. If $\left(\xi_{x}^{*} ; y_{x}^{*}\right) \in \Omega(x)$, then we have

$$
y_{x}^{*}=\min \left\{\pi\left(\xi_{x}^{*}\right), u\left(\xi_{x}^{*}, x\right)\right\} .
$$

Proof If $\left(\xi_{x}^{*} ; y_{x}^{*}\right)$ is a global maximizer of $(21)$ for any $x \in X$, then we have either $(22)$ or $y_{x}^{*}<\min \left\{\pi\left(\xi_{x}^{*}\right), u\left(\xi_{x}^{*}, x\right)\right\}$. In fact, $y_{x}^{*}<\min \left\{\pi\left(\xi_{x}^{*}\right), u\left(\xi_{x}^{*}, x\right)\right\}$ does not hold. The reason is as follows. If $y_{x}^{*}<\min \left\{\pi\left(\xi_{x}^{*}\right), u\left(\xi_{x}^{*}, x\right)\right\}$ holds, setting $\bar{y}_{x}:=\frac{1}{2}\left(y_{x}^{*}+\min \left\{\pi\left(\xi_{x}^{*}\right), u\left(\xi_{x}^{*}, x\right)\right\}\right)$, then we have $\bar{y}_{x}<\min \left\{\pi\left(\xi_{x}^{*}\right), u\left(\xi_{x}^{*}, x\right)\right\}$. Thus, we have $\bar{y}_{x}<\pi\left(\xi_{x}^{*}\right)$ and $\bar{y}_{x}<u\left(\xi_{x}^{*}, x\right)$, that is, $\left(\xi_{x}^{*} ; \bar{y}_{x}\right)$ is feasible for the problem (21). In addition, it is easy to verify that $\bar{y}_{x}>y_{x}^{*}$ if $y_{x}^{*}<$ $\min \left\{\pi\left(\xi_{x}^{*}\right), u\left(\xi_{x}^{*}, x\right)\right\}$. Clearly, this conflicts with the assumption that $\left(\xi_{x}^{*} ; y_{x}^{*}\right)$ is a global maximizer of (21). Thus, only (22) holds.

Lemma 2 shows that the optimal solutions of the lower level problem (18) and the problem (21) are equivalent globally. In other words, (21) is an equivalent reformulation of the problem (18). In the following, we focus on the BLPP (17) with the reformulated lower level problem (21). For any given $x \in X$, we can understand from (13) and (14) that $\pi(\cdot)$ is a differentiable concave function and $u(\cdot, x)$ is an affine function, respectively. Hence, (21) is a smooth convex optimization problem. Due to $\xi_{l}<\xi_{u}$, it is easy to verify that the Slater's condition holds for the problem (21) for any given $x \in X$. The common method to solve a BLPP with a convex lower level problem is to translate it into an MPEC by replacing the lower level problem with its Karush-KuhnTucker (KKT) condition (e.g., [3,22]). Based on the KKT method, we know that solving (21) is equivalent to solving its KKT condition, that is, solving 
the following system with equalities and inequalities:

$$
\left\{\begin{array}{l}
(1-w) \pi^{\prime}(\xi)+w u_{\xi}^{\prime}(\xi, x)+\alpha-\beta=0 \\
0 \leq 1-w \perp \pi(\xi)-y \geq 0 \\
0 \leq w \perp u(\xi, x)-y \geq 0 \\
0 \leq \alpha \perp \xi-\xi_{l} \geq 0 \\
0 \leq \beta \perp \xi_{u}-\xi \geq 0
\end{array}\right.
$$

where $w \in R, \alpha, \beta \in R^{n}$ and $0 \leq a \perp b \geq 0$ is called the complementarity constraint, which means that $a \geq 0, b \geq 0$ and $a^{T} b=0$. Setting $H_{1}(w, \alpha, \beta):=(1-w ; w ; \alpha ; \beta), H_{2}(x, \xi, y):=\left(\pi(\xi)-y ; u(\xi, x)-y ; \xi-\xi_{l} ; \xi_{u}-\xi\right)$ and $h(x, \xi, w, \alpha, \beta):=(1-w) \pi^{\prime}(\xi)+w u_{\xi}^{\prime}(\xi, x)+\alpha-\beta$, we can reformulate Model A as the following MPEC:

$$
\begin{array}{cl}
\max _{z} & \xi^{T} x \\
\text { s.t. } & A x \leq b, x \geq 0, h(x, \xi, w, \alpha, \beta)=0, \\
& 0 \leq H_{1}(w, \alpha, \beta) \perp H_{2}(x, \xi, y) \geq 0, \\
& z=(x ; \xi ; w ; \alpha ; \beta) \in R^{n} \times R^{n} \times R \times R^{n} \times R^{n} .
\end{array}
$$

Although (24) is a single-level optimization problem, solving it is still difficult. First, (24) is not a convex optimization problem due to the existence of complementarity constraints. Second, (24) fails to satisfy the general constraint qualifications, such as Mangasarian-Fromovizt constraint qualification (MFCQ) which can ensure that the local minimizer must be a stationary point. It is not difficult to understand that the above MPEC has a combinatorial structure, that is, its feasible region is a union of lots of pieces. It means that it is inappropriate to utilize classical nonlinear optimization theories and algorithms to solve $(24)$ directly $[42,43]$. To deal with this special nonlinear optimization problem, various theories and methods on MPECs have been made by researches (e.g., $[28,38,52])$. However, all these methods need strong assumptions and can only find out stationary points. Another appealing way to the BLPP is based on the so-called optimal value function of the lower level problem. This approach was first introduced by Outrata [49] for obtaining a numerical solution and subsequently used by Ye and Zhu [67] for obtaining necessary optimality conditions. Although this approach does not require the convexity assumption of the lower level problem, solving the reformulation is significantly difficult because it is neither smooth nor convex, and what is more, it is quite complicated to design effective numerical algorithms to solve the reformulation because it consists of an implicitly determined constraint function; see, e.g., $[23,42,66]$. Due to their inherent mathematical difficulties, so far most references handling a BLPP assume that the lower level is a convex optimization problem and then solve its KKT reformulation.

Without using the traditional KKT reformulation, we propose new approaches to solve the ODPPMs (Model A and Model B). First, we consider the following two single-level optimization problems: 


\section{Model I:}

$$
\max _{(x ; \xi)} \xi^{T} x \quad \text { s.t. } A x \leq b, x \geq 0, \xi \in\left[\xi_{l}, \xi_{u}\right], F(\xi, x) \leq 0,
$$

where

$$
F(\xi, x):=u(\xi, x)-\pi(\xi)
$$

Model II:

$$
\begin{array}{cl}
\max _{z} & L(x, \xi, \lambda, \eta, \tau) \\
\text { s.t. } & A x \leq b, x \geq 0, \xi \in\left[\xi_{l}, \xi_{u}\right], G(x, \xi, \lambda, \eta, \tau)=0, \\
& z=(x ; \xi ; \lambda ; \eta ; \tau) \in R^{n} \times R^{n} \times R_{+}^{n} \times R_{+}^{n} \times R_{+},
\end{array}
$$

where

$$
\begin{gathered}
L(x, \xi, \lambda, \eta, \tau):=(1-\tau) u(\xi, x)+\tau(1-\pi(\xi))+\lambda^{T}\left(\xi-\xi_{u}\right)+\eta^{T}\left(\xi_{l}-\xi\right), \\
G(x, \xi, \lambda, \eta, \tau):=(1-\tau) u_{\xi}^{\prime}(\xi, x)-\tau \pi^{\prime}(\xi)+\lambda-\eta
\end{gathered}
$$

In what follows, we examine the relationship between Model I and Model A and the relationship between Model II and Model B.

Theorem 1 Suppose that $\left(x^{*} ; \xi^{*}\right)$ is a global optimal solution of Model I, then it is still global optimal for Model $A$.

Proof Suppose that $\xi(x)$ is a global optimal solution of the lower level problem (18) where $x \in X$, then we have

$$
\min \{\pi(\xi(x)), u(\xi(x), x)\} \geq \min \{\pi(\xi), u(\xi, x)\}, \quad \forall \xi \in\left[\xi_{l}, \xi_{u}\right] .
$$

In fact, $\xi(x)$ must satisfy the following condition:

$$
u(\xi(x), x)-\pi(\xi(x)) \leq 0
$$

The reason is as follows.

If (31) does not hold, then we have $\pi(\xi(x))=\min \{\pi(\xi(x)), u(\xi(x), x)\}$. Considering (30), if $\pi(\xi)<u(\xi, x)$, we can obtain that

$$
\pi(\xi(x))=\min \{\pi(\xi(x)), u(\xi(x), x)\} \geq \min \{\pi(\xi), u(\xi, x)\}=\pi(\xi) .
$$

Clearly, if $\pi(\xi(x))<u(\xi(x), x)$, then $\pi(\xi)$ is still global optimal for the following optimization problem:

$$
\max _{\xi} \pi(\xi) \quad \text { s.t. } \pi(\xi)-u(\xi, x)<0, \xi \in\left[\xi_{l}, \xi_{u}\right]
$$

From (13), we know that $\pi(\cdot)$ is a concave function and there exists $\mu \in\left[\xi_{l}, \xi_{u}\right]$ satisfying $\pi(\mu)=\max \left\{\pi(\xi): \xi \in\left[\xi_{l}, \xi_{u}\right]\right\}=1$. Together with $\pi(\xi) \in[0,1]$ and $u(\xi, x) \in[0,1]$, we can obtain that

$$
\pi(\mu)-u(\mu, x)=1-u(\mu, x) \geq 0 .
$$


It is clear that $\xi(x) \neq \mu$ if $\pi(\xi(x))<u(\xi(x), x)$. Since $\pi(\cdot)$ given by (13) is concave and $u(\cdot, x)$ given by (14) is affine, there must exist $\xi_{x}=(1-\sigma) \mu+$ $\sigma \xi(x) \in\left[\xi_{l}, \xi_{u}\right]$ where $0<\sigma<1$ such that

$$
\pi\left(\xi_{x}\right)>\pi(\xi(x)) \text { and } \pi\left(\xi_{x}\right)-u\left(\xi_{x}, x\right)<0,
$$

which conflict with the assumption that $\xi(x)$ is the global optimal solution of (32). Hence, (31) is true. Thus, we can rewrite (18) as the following optimization problem:

$$
\max _{\xi} u(\xi, x) \quad \text { s.t. } u(\xi, x)-\pi(\xi) \leq 0, \xi \in\left[\xi_{l}, \xi_{u}\right] .
$$

Since $u(\xi, x)$ is the normalized profit function, we know that solving Model A is equivalent to solving the following single-level optimization problem:

$$
\max _{(x ; \xi)} \xi^{T} x \quad \text { s.t. } A x \leq b, x \geq 0, \xi \in\left[\xi_{l}, \xi_{u}\right], u(\xi, x)-\pi(\xi) \leq 0 .
$$

Using (26), we can rewrite (33) as Model I. Clearly, if $\left(x^{*} ; \xi^{*}\right)$ is a global optimal solution of Model I, then it is still global optimal for Model A.

Theorem 2 Suppose that $\left(x^{*} ; \xi^{*} ; \lambda^{*} ; \eta^{*} ; \tau^{*}\right)$ is a global optimal solution of Model II, then $\left(x^{*} ; \xi^{*}\right)$ is global optimal for Model B.

Proof Suppose that $\xi(x)$ is a global optimal solution of the lower level problem (20) where $x \in X$, then we have

$$
\max \{1-\pi(\xi(x)), u(\xi(x), x)\} \leq \max \{1-\pi(\xi), u(\xi, x)\}, \quad \forall \xi \in\left[\xi_{l}, \xi_{u}\right] .
$$

We divide the difference of $1-\pi(\xi(x))$ and $u(\xi(x), x)$ into the following two cases:

$$
1-\pi(\xi(x))-u(\xi(x), x)>0 \text { and } 1-\pi(\xi(x))-u(\xi(x), x) \leq 0 .
$$

In fact, the first case does not hold. The reason is as follows.

If the first case holds, that is, $\pi(\xi(x))<1-u(\xi(x), x)$, then we have

$$
1-\pi(\xi(x))=\max \{1-\pi(\xi(x)), u(\xi(x), x)\} \leq \max \{1-\pi(\xi), u(\xi, x)\},
$$

which implies that $\xi(x)$ is still global optimal for the following optimization problem:

$$
\min _{\xi} 1-\pi(\xi) \quad \text { s.t. } 1-\pi(\xi)>u(\xi, x), \xi \in\left[\xi_{l}, \xi_{u}\right] .
$$

From (13), we know that $\pi(\cdot)$ is a concave function and there exists $\mu \in\left[\xi_{l}, \xi_{u}\right]$ satisfying $\pi(\mu)=\max \left\{\pi(\xi): \xi \in\left[\xi_{l}, \xi_{u}\right]\right\}=1$. Together with $\pi(\xi) \in[0,1]$ and $u(\xi, x) \in[0,1]$, we have that

$$
1-\pi(\mu)=0 \leq u(\xi(x), x) .
$$


Clearly, $\xi(x) \neq \mu$ if $1-\pi(\xi(x))>u(\xi(x), x)$. Since $\pi(\cdot)$ given by (13) is concave and $u(\cdot, x)$ given by (14) is affine, there must exist $\xi_{x}=(1-\sigma) \mu+\sigma \xi(x) \in$ $\left[\xi_{l}, \xi_{u}\right]$ where $0<\sigma<1$ such that

$$
1-\pi\left(\xi_{x}\right)<1-\pi(\xi(x)) \text { and } 1-\pi\left(\xi_{x}\right)>u\left(\xi_{x}, x\right)
$$

which conflict with the assumption that $\xi(x)$ is the global optimal solution of (34). Therefore, solving (20) is equivalent to solving the following optimization problem:

$$
\min _{\xi} u(\xi, x) \quad \text { s.t. } 1-\pi(\xi)-u(\xi, x) \leq 0, \xi \in\left[\xi_{l}, \xi_{u}\right]
$$

Since $u(\xi, x)$ is the normalized profit function, we can rewrite Model B as the following max-min optimization problem:

$$
\max _{x} \min _{\xi}\left\{u(\xi, x): 1-\pi(\xi)-u(\xi, x) \leq 0, \xi \in\left[\xi_{l}, \xi_{u}\right], A x \leq b, x \geq 0\right\} .
$$

From (13) and (14), we know that solving (35) is to solve a convex optimization problem since $u(\cdot, x)$ is affine for any $x \neq 0$ and $1-\pi(\cdot)-u(\cdot, x)$ is convex. Since $1-\pi(\cdot)-u(\cdot, x)$ is a convex function, it is easy to check that there must exist $\xi_{0} \in\left[\xi_{l}, \xi_{u}\right]$ such that

$$
1-\pi\left(\xi_{0}\right)-u\left(\xi_{0}, x\right)<0 \text { and } \xi_{l}<\xi_{0}<\xi_{u},
$$

which implies that Slater's constraint qualification holds for the problem (35). From the strongly dual theory for a convex optimization problem [58, Section 5.2 ], we know that solving (35) can be equivalent to solving its duality problem and they have the same optimal value. Let us give the Lagrange function of the problem (35) as follows:

$$
\begin{aligned}
& L(x, \xi, \lambda, \eta, \tau) \\
& \quad:=u(\xi, x)+\lambda^{T}\left(\xi-\xi_{u}\right)+\eta^{T}\left(\xi_{l}-\xi\right)+\tau(1-\pi(\xi)-u(\xi, x)),
\end{aligned}
$$

where $\lambda \in R_{+}^{n}, \eta \in R_{+}^{n}$ and $\tau \in R_{+}$. Clearly, $L(x, \xi, \lambda, \eta, \tau)$ is convex for the variable $\xi$ for any fixed $x \in X, \lambda \geq 0, \eta \geq 0$ and $\tau \geq 0$. Thus, we can express its dual problem as

$$
\max _{(\lambda ; \eta ; \tau)} L(x, \xi, \lambda, \eta, \tau) \text { s.t. } G(x, \xi, \lambda, \eta, \tau)=0, \lambda, \eta \in R_{+}^{n}, \tau \in R_{+},
$$

where

$$
G(x, \xi, \lambda, \eta, \tau)=L_{\xi}^{\prime}(x, \xi, \lambda, \eta, \tau) .
$$

(38) is also called the Wolfe duality problem. We can further rewrite (37) as (28), (39) as (29). Since the strongly dual condition holds for the problem (35), we know that solving (36) is equivalent to solving Model II. Clearly, if $\left(x^{*} ; \xi^{*} ; \lambda^{*} ; \eta^{*} ; \tau^{*}\right)$ is a global optimal solution of Model II, then $\left(x^{*} ; \xi^{*}\right)$ is global optimal for Model B. 
Comments: There are two papers $[69,70]$ related to this research. In the paper [69], we propose a production planning model with the one-shot decision theory in which only the formula (15) is considered to obtain the solution of the lower level problem. That is, the model in the paper [69] is a special case of the model (11) proposed in this research. Although the model in the paper [69] is the same as Model A in this research, we propose completely different approaches to solve them. The approach proposed in the paper [69] is a KKT-based method. In order to replace the lower level problem by the KKT condition, we utilize the smooth function to approximate the min function in the lower level problem and assume that the functions involved in the lower level are all twice continuously differentiable and concave. Clearly, such assumptions are stricter than the ones in this paper and the approach proposed in the paper [69] can only obtain approximation solutions of Model A. Further, as mentioned in Section 3, the KKT reformulation is very difficult to be solved because of the existence of complementarity constraints. On the contrary, the proposed approaches to Models A and B in this paper are not KKT-based and can solve Models A and B easily. In the paper [70], we propose several approaches to bilevel programming problems with max-min or min-max lower level programs; these approaches are applicable for only the case that all decision variables are one-dimensional, and no constraint exists. Hence, Models A and $\mathrm{B}$ in this research cannot be solved by the proposed methods in the paper $[70]$.

\section{Numerical examples and computational discussion}

In order to illustrate the proposed approaches, let us consider a numerical example as follows. An apparel manufacturer is planning to produce four types of new fashion clothes for the coming summer season. For producing fashion clothes 1, 2, 3 and 4, four kinds of resources, that is, A, B, C and D are needed. The available amounts of A, B, C and D are 1500, 2250, 1100 and 1300 units, respectively. The amounts of resource A needed for producing one unit fashion clothes 1, 2, 3 and 4 are 2, 3, 3 and 2 units, respectively; the amounts of resource $\mathrm{B}$ needed for producing one unit fashion clothes $1,2,3$ and 4 are 2, 3, 4 and 5 units, respectively; the amounts of resource $\mathrm{C}$ needed for producing one unit fashion clothes 1,2, 3 and 4 are 3,2, 2 and 1 units, respectively; the amounts of resource $\mathrm{D}$ needed for producing one unit fashion clothes 1, 2, 3 and 4 are 1,2, 2 and 3 units, respectively. Hence, the feasible set of production levels is

$$
X=\left\{\begin{array}{l}
2 x_{1}+3 x_{2}+3 x_{3}+2 x_{4} \leq 1500 \\
2 x_{1}+3 x_{2}+4 x_{3}+5 x_{4} \leq 2250 \\
3 x_{1}+2 x_{2}+2 x_{3}+x_{4} \leq 1100 \\
x_{1}+2 x_{2}+2 x_{3}+3 x_{4} \leq 1300
\end{array}\right\}
$$

The uncertainty of the profits per unit for clothes $1,2,3$ and 4 is characterized by the truncated normal distribution where the mean vector is $\mu$, the 
covariance matrix is $\Sigma$ (a positive definite matrix) and the support is set as

$$
\Omega:=[\mu-k \sigma, \mu+k \sigma] \text { with } k>0
$$

where $\sigma_{i}:=\sqrt{\Sigma_{i i}}$ represents the standard deviation of $\Xi_{i}$ for $i=1,2,3,4$. In particular, the relative likelihood function (13) can be given as

$$
\pi(\xi)=1-(\xi-\mu)^{T} \frac{\Sigma^{-1}}{k^{2}\left(\sigma^{T} \Sigma^{-1} \sigma\right)}(\xi-\mu) .
$$

Since $\Sigma^{-1}$ is a positive definite matrix, $\pi(\xi)$ given by (40) is a quadratic concave function which means that the mean vector has the maximal relative likelihood degree 1 and the vector which has a longer Euclidean distance from the mean vector will have a less relative likelihood degree. By (14), we have

$$
u(\xi, x)=\frac{\xi^{T} x-v_{l}}{v_{u}-v_{l}},
$$

where $v_{l}=\min \left\{(\mu-k \sigma)^{T} x: x \in X\right\}$ and $v_{u}=\max \left\{(\mu+k \sigma)^{T} x: x \in X\right\}$.

The expected profit based production planning model (3) becomes

$$
\max _{x} \mu^{T} x \quad \text { s.t. } x \in X .
$$

The max-max approach based production planning model (6) becomes

$$
\max _{x}(\mu+k \sigma)^{T} x \quad \text { s.t. } x \in X .
$$

The max-min approach based production planning model (8) becomes

$$
\max _{x}(\mu-k \sigma)^{T} x \quad \text { s.t. } x \in X .
$$

In the following, we utilize the interior-point algorithm from Global Optimization Toolbox of MATLAB 7.10 to solve Models A and B, and use the simplex algorithm to solve the other traditional optimization models (42), (43) and (44). The numerical results and analysis are given in the following sections.

4.1 Numerical results

Case I: $k=2, \Sigma=\Sigma_{0}$ and $\mu=\mu_{0}$ where

$$
\Sigma_{0}=\left(\begin{array}{llll}
2500 & 1250 & 1250 & 1250 \\
1250 & 2500 & 1250 & 1250 \\
1250 & 1250 & 2500 & 1250 \\
1250 & 1250 & 1250 & 2500
\end{array}\right) \text { and } \mu_{0}=\left(\begin{array}{c}
150 \\
200 \\
200 \\
150
\end{array}\right) \text {. }
$$

We obtain the results shown in Table 1. 
Table 1 Solutions of Production Planning Models for Case I

\begin{tabular}{|c|c|c|c|c|c|}
\hline Model & $\xi^{*}$ & $x^{*}$ & $\pi\left(\xi^{*}\right)$ & $u\left(\xi^{*}, x^{*}\right)$ & $\xi^{* T} x^{*}$ \\
\hline (43) & $\left(\begin{array}{l}250.0000 \\
300.0000 \\
300.0000 \\
250.0000\end{array}\right)$ & $\left(\begin{array}{l}100.0000 \\
200.0000 \\
100.0000 \\
200.0000\end{array}\right)$ & 0.0000 & 1.0000 & 165000 \\
\hline A & $\left(\begin{array}{l}191.5182 \\
244.4839 \\
244.4839 \\
197.4494\end{array}\right)$ & $\left(\begin{array}{l}100.0000 \\
150.0000 \\
150.0000 \\
200.0000\end{array}\right)$ & 0.7999 & 0.7999 & 131990 \\
\hline (42) & $\left(\begin{array}{l}150.0000 \\
200.0000 \\
200.0000 \\
150.0000\end{array}\right)$ & $\left(\begin{array}{l}100.0000 \\
186.1648 \\
113.8352 \\
200.0000\end{array}\right)$ & 1.0000 & 0.6364 & 105000 \\
\hline B & $\left(\begin{array}{c}91.9217 \\
133.4775 \\
133.4775 \\
88.3976\end{array}\right)$ & $\left(\begin{array}{c}84.9330 \\
180.1356 \\
180.1324 \\
124.6650\end{array}\right)$ & 0.5945 & 0.4055 & 66915 \\
\hline (44) & $\left(\begin{array}{c}50.0000 \\
100.0000 \\
100.0000 \\
50.0000\end{array}\right)$ & $\left(\begin{array}{c}0.0000 \\
236.7627 \\
263.2373 \\
0.0000\end{array}\right)$ & 0.0000 & 0.3030 & 50000 \\
\hline
\end{tabular}

For examining how the solutions change with $k$ for Case I, we consider the following case.

Case II: $k=3, \Sigma=\Sigma_{0}$ and $\mu=\mu_{0}$.

The obtained results are shown in Table 2 . 
Table 2 Solutions of Production Planning Models for Case II

\begin{tabular}{|c|c|c|c|c|c|}
\hline Model & $\xi^{*}$ & $x^{*}$ & $\pi\left(\xi^{*}\right)$ & $u\left(\xi^{*}, x^{*}\right)$ & $\xi^{* T} x^{*}$ \\
\hline (43) & $\left(\begin{array}{l}300.0000 \\
350.0000 \\
350.0000 \\
300.0000\end{array}\right)$ & $\left(\begin{array}{c}100.0000 \\
202.1351 \\
97.8649 \\
200.0000\end{array}\right)$ & 0.0000 & 1.0000 & 195000 \\
\hline A & $\left(\begin{array}{l}213.5324 \\
281.6845 \\
254.4564 \\
222.6085\end{array}\right)$ & $\left(\begin{array}{c}100.0000 \\
300.0000 \\
0.0000 \\
200.0000\end{array}\right)$ & 0.7712 & 0.7712 & 150380 \\
\hline$(42)$ & $\left(\begin{array}{l}150.0000 \\
200.0000 \\
200.0000 \\
150.0000\end{array}\right)$ & $\left(\begin{array}{l}100.0000 \\
186.1648 \\
113.8352 \\
200.0000\end{array}\right)$ & 1.0000 & 0.5385 & 105000 \\
\hline B & $\left(\begin{array}{c}77.1232 \\
113.8610 \\
113.8608 \\
75.6643\end{array}\right)$ & $\left(\begin{array}{c}78.1756 \\
193.6479 \\
193.6497 \\
90.8779\end{array}\right)$ & 0.7077 & 0.2923 & 57003 \\
\hline (44) & $\left(\begin{array}{c}0.0000 \\
50.0000 \\
50.0000 \\
0.0000\end{array}\right)$ & $\left(\begin{array}{c}0.0000 \\
271.1877 \\
228.8123 \\
0.0000\end{array}\right)$ & 0.0000 & 0.1282 & 25000 \\
\hline
\end{tabular}

We examine how the solutions change with $\Sigma$ for Case I. We consider the following case III.

Case III: $k=2, \Sigma=1.44 \times \Sigma_{0}$ and $\mu=\mu_{0}$.

The obtained results are shown in Table 3 . 
Table 3 Solutions of Production Planning Models for Case III

\begin{tabular}{|c|c|c|c|c|c|}
\hline Model & $\xi^{*}$ & $x^{*}$ & $\pi\left(\xi^{*}\right)$ & $u\left(\xi^{*}, x^{*}\right)$ & $\xi^{* T} x^{*}$ \\
\hline (43) & $\left(\begin{array}{l}270.0000 \\
320.0000 \\
320.0000 \\
270.0000\end{array}\right)$ & $\left(\begin{array}{l}100.0000 \\
200.0000 \\
100.0000 \\
200.0000\end{array}\right)$ & 0.0000 & 1.0000 & 177000 \\
\hline A & $\left(\begin{array}{l}201.8225 \\
255.5241 \\
255.5241 \\
209.2257\end{array}\right)$ & $\left(\begin{array}{l}100.0000 \\
150.0000 \\
150.0000 \\
200.0000\end{array}\right)$ & 0.7835 & 0.7835 & 138680 \\
\hline$(42)$ & $\left(\begin{array}{l}150.0000 \\
200.0000 \\
200.0000 \\
150.0000\end{array}\right)$ & $\left(\begin{array}{l}100.0000 \\
186.1648 \\
113.8352 \\
200.0000\end{array}\right)$ & 1.0000 & 0.5932 & 105000 \\
\hline B & $\left(\begin{array}{c}85.3928 \\
124.8230 \\
124.8230 \\
82.7798\end{array}\right)$ & $\left(\begin{array}{c}81.5170 \\
186.9661 \\
186.9659 \\
107.5849\end{array}\right)$ & 0.6467 & 0.3533 & 62542 \\
\hline (44) & $\left(\begin{array}{l}30.0000 \\
80.0000 \\
80.0000 \\
30.0000\end{array}\right)$ & $\left(\begin{array}{c}0.0000 \\
258.4314 \\
241.5686 \\
0.0000\end{array}\right)$ & 0.0000 & 0.2260 & 40000 \\
\hline
\end{tabular}

Table 4 The scenarios associated with the optimal solutions in Cases I and II

\begin{tabular}{cccccc}
\hline & Model (43) & Model A & Model (42) & Model B & Model (44) \\
\hline I & $\left(\begin{array}{l}250.0000 \\
300.0000 \\
300.0000 \\
250.0000\end{array}\right)$ & $\left(\begin{array}{l}191.5182 \\
244.4839 \\
244.4839 \\
197.4494\end{array}\right)$ & $\left(\begin{array}{l}150.0000 \\
200.0000 \\
200.0000 \\
150.0000\end{array}\right)$ & $\left(\begin{array}{c}91.9217 \\
133.4775 \\
133.4775 \\
88.3976\end{array}\right)$ & $\left(\begin{array}{c}50.0000 \\
100.0000 \\
100.0000 \\
50.0000\end{array}\right)$ \\
\hline II & $\left(\begin{array}{l}300.0000 \\
350.0000 \\
350.0000 \\
300.0000\end{array}\right)$ & $\left(\begin{array}{l}213.5324 \\
281.6845 \\
254.4564 \\
222.6085\end{array}\right)$ & $\left(\begin{array}{l}150.0000 \\
200.0000 \\
200.0000 \\
150.0000\end{array}\right)$ & $\left(\begin{array}{c}77.1232 \\
113.8610 \\
113.8608 \\
75.6643\end{array}\right)$ & $\left(\begin{array}{c}0.0000 \\
50.0000 \\
50.0000 \\
0.0000\end{array}\right)$ \\
\hline
\end{tabular}


Table 5 The scenarios associated with the optimal solutions in Cases I and III

\begin{tabular}{cccccc}
\hline & Model (43) & Model A & Model (42) & Model B & Model (44) \\
\hline \multirow{4}{*}{ I } & $\left(\begin{array}{l}250.0000 \\
300.0000 \\
300.0000 \\
250.0000\end{array}\right)$ & $\left(\begin{array}{l}191.5182 \\
244.4839 \\
244.4839 \\
197.4494\end{array}\right)$ & $\left(\begin{array}{l}150.0000 \\
200.0000 \\
200.0000 \\
150.0000\end{array}\right)$ & $\left(\begin{array}{c}91.9217 \\
133.4775 \\
133.4775 \\
88.3976\end{array}\right)$ & $\left(\begin{array}{c}50.0000 \\
100.0000 \\
100.0000 \\
50.0000\end{array}\right)$ \\
\hline \multirow{2}{*}{ III } & $\left(\begin{array}{l}270.0000 \\
320.0000 \\
320.0000 \\
270.0000\end{array}\right)$ & $\left(\begin{array}{l}201.8225 \\
255.5241 \\
255.5241 \\
209.2257\end{array}\right)$ & $\left(\begin{array}{l}150.0000 \\
200.0000 \\
200.0000 \\
150.0000\end{array}\right)$ & $\left(\begin{array}{c}85.3928 \\
124.8230 \\
124.8230 \\
82.7798\end{array}\right)$ & $\left(\begin{array}{c}30.0000 \\
80.0000 \\
80.0000 \\
30.0000\end{array}\right)$ \\
\hline
\end{tabular}

Table 6 The optimal total profit for Cases I and II

\begin{tabular}{cccccc}
\hline & Model (43) & Model A & Model (42) & Model B & Model (44) \\
\hline I & 165000 & 131990 & 105000 & 66915 & 50000 \\
\hline II & 195000 & 150380 & 105000 & 57003 & 25000 \\
\hline
\end{tabular}

Table 7 The optimal total profit for Cases I and III

\begin{tabular}{cccccc}
\hline & Model (43) & Model A & Model (42) & Model B & Model (44) \\
\hline I & 165000 & 131990 & 105000 & 66915 & 50000 \\
\hline III & 177000 & 138680 & 105000 & 62542 & 40000 \\
\hline
\end{tabular}

Let us give an explanation for the above numerical examples. Model $\mathrm{A}$ in Table 1 shows that the optimal production level for an active manufacturer. The active optimal production level is $(100.0000 ; 150.0000 ; 150.0000 ; 200.0000)$ and its focus point (scenario) is $(191.5182 ; 244.4839 ; 244.4839 ; 197.4494)$; the relative likelihood degree of this scenario is 0.7999 ; when this scenario occurs, the satisfaction level of this production level is 0.7999. In other words, the reason that an active manufacturer chooses the production level (100.0000; $150.0000 ; 150.0000 ; 200.0000)$ is that the scenario $(191.5182 ; 244.4839 ; 244.4839$; 197.4494) is the most appropriate for this manufacturer. Model B in Table 1 shows that the optimal production level for a passive manufacturer. The passive optimal production level is $(84.9330 ; 180.1356 ; 180.1324 ; 124.6650)$ and its focus point (scenario) is $(91.9217 ; 133.4775 ; 133.4775 ; 88.3976)$; the relative likelihood degree of this scenario is 0.5945 ; when the scenario occurs, the satisfaction level of this production level is 0.4055 . It means that the scenario $(91.9217 ; 133.4775 ; 133.4775 ; 88.3976)$ is the most acceptable scenario amongst all unfavorable ones, a passive manufacturer chooses the production 
level $(84.9330 ; 180.1356 ; 180.1324 ; 124.6650)$ based on this scenario. Model (42) in Table 1 corresponds to the expected profit maximization manufacturer where the mean of profits per units of four products is $(150 ; 200 ; 200 ; 150)$ whose relative likelihood degree is 1 and it can lead to the satisfaction level of 0.6364 for the optimal production level. Models (43) and (44) in Table 1 are for the max-max manufacturer and the max-min manufacturer, respectively. The max-max manufacturer takes into account the upper bound of profits per units of four products $(250 ; 300 ; 300 ; 250)$ whereas the max-min manufacturer considers the lower bound of profits per units of four products $(50 ; 100 ; 100$; $50)$.

Table 2 shows how the optimal solutions change with the varying of the feasible region of the realization of random variables, that is, the varying of $k$; Table 3 shows how the optimal solutions change with the varying of uncertainty, that is, the varying of $\Sigma$. From these tables, we know different models provide different optimal solutions which can reflect different consideration for handling the uncertainty. However, as summarized in Tables 4 and 5, in all cases, the profit per unit of each product which is taken into account for obtaining the optimal production level will increase according to the order of the max-min manufacturer, the passive manufacturer, the expected profit maximization manufacturer, the active manufacturer and the max-max manufacturer. In addition, Tables 6 and 7 show that the profit anticipated by a max-max manufacturer is larger than the one by an active manufacturer; the profit anticipated by an active manufacturer is larger than the one by an expected profit maximization manufacturer; the profit anticipated by an expected profit maximization manufacturer is larger than the one by a passive manufacturer; the profit anticipated by a passive manufacturer is larger than the one by a max-min manufacturer. It means that the max-max manufacturer is the most optimistic, the max-min manufacturer is the most pessimistic, the expected profit maximization manufacturer is at the middle of the active and passive manufacturers.

When we compare the profit per unit of each product associated with the optimal production levels between Case I and Case II (shown in Table 4), we can find that increasing the feasible set of the realization of random variables, that is, $k$ increasing from 2 (Case I) to 3 (Case II), the max-min manufacturer (Model (44)) and the passive manufacturer (Model B) will take a more conservative attitude so that the profit per unit of each product associated with the optimal production level will decrease accordingly; on the contrary, the max-max manufacturer ((Model (43)) and the active manufacturer (Model A) will take a more aggressive attitude so that the profit per unit of each product associated with the optimal production level will increase accordingly; however, an expected profit maximization manufacturer only takes into account the mean vector of the profits per unit of products.

When we compare the profit per unit of each product associated with the optimal production levels between Case I and Case III (shown in Table 5), we can find that increasing the uncertainty of profits, that is, changing $\Sigma$ from $\Sigma_{0}$ (Case I) to $1.44 \times \Sigma_{0}$ (Case III), will cause the max-min manufacturer (Model 
(44)) and the passive manufacturer (Model B) more vigilant so that the profit per unit of each product associated with the optimal production level will decrease accordingly; on the contrary, the max-max manufacturer (Model (43)) and the active manufacturer (Model A) will become more optimistic so that the profit per unit of each product associated with the optimal production level will increase accordingly; however, the action of an expected profit maximization manufacturer will remain unchanged.

The obtained managerial insights are intuitively acceptable and can be used as a sort of criterion for selecting a production planning model to fit the preference of the different types of decision makers.

\section{Conclusions}

We propose a new production planning model for a manufacturer that is planning to produce multiple innovative products with short life-cycles. Different from the existing production planning models, we build the one-shot decision theory based production planning models in which the optimal production quantities are obtained based on the scenarios which are the most appropriate for the manufacturer with considering the profit and the probability. In addition, the relationships between the proposed models and the existing ones has been clarified. In order to facilitate the understanding of the proposed models, the production planning problem is confined to the basic one. However, the research provides a fundamentally new idea for solving the production planning problem under uncertainty; the proposed models can easily be extended into complicated ones by adding more detailed and realistic constraints.

Since the proposed models are the bilevel programming problems, which are difficult to be solved. We propose two approaches to solve them by translating them into general single-level optimization problems so that they can be solved with the commonly used optimization methods and software. It should be emphasized that the research shows an alternative for solving the bilevel programming problems instead of using KKT-based methods. As an extension of this research, we will apply the proposed models to real-world production planning problems in the near future.

Acknowledgements This research was supported by JSPS KAKENHI Grant Number 15K03599.

\section{Compliance with ethical standards}

Conflict of interest: We declare that no conflict of interest exists in this paper. 


\section{References}

1. Alem, D.J., \& Morabito, R. Production planning in furniture settings via robust optimization. Computers \& Operations Research, 39(2), 139-150, 2012.

2. Allais, M. Le Comportement de l'homme rationnel devant le risque: critique des postulats et axiomes de l'ecole americaine. Econometrica, 21(4), 503-546, 1953.

3. Allende, G.B., \& Still, G. Solving bilevel programs with the KKT-approach. Mathematical Programming, 138(1-2), 309-332, 2013.

4. Alvarez, P.P., \& Vera, J.R. Application of robust optimization to the sawmill planning problem. Annals of Operations Research, 219(1), 457-475, 2014.

5. Aouam, T., \& Brahimi, N. Integrated production planning and order acceptance under uncertainty: A robust optimization approach. European Journal of Operational Research, 228(3), 504-515, 2013.

6. Ardjmand, E., Weckman, G.R., Young II, W.A., Sanei Bajgiran, O., \& Aminipour, B. A robust optimisation model for production planning and pricing under demand uncertainty. International journal of production research, 54(13), 3885-3905, 2016.

7. Bagnoli M., \& Bergstrom T. Log-concave probability and its applications. Economic theory, 26(2), 445-469, 2005.

8. Bard, J.F. Some properties of the bilevel programming problem. Journal of Optimization Theory and Applications, 68(2), 371-378, 1991.

9. Ben-Tal, A., Goryashko, A., Guslitzer, E., \& Nemirovski, A. Adjustable robust solutions of uncertain linear programs. Mathematical Programming, 99(2), 351-376, 2004.

10. Ben-Tal, A., El Ghaoui, L., \& Nemirovski, A. Robust optimization. Princeton University Press, 2009.

11. Birge, J.R., \& Louveaux, F. Introduction to stochastic programming. Springer-Verlag, New York, 1997.

12. Bordalo, P., Gennaioli, N., \& Shleifer, A. Salience theory of choice under risk. Quarterly Journal of Economics, 127(3), 1243-1285, 2012.

13. Bornapour, M., \& Hooshmand, R.A. An efficient scenario-based stochastic programming for optimal planning of combined heat, power, and hydrogen production of molten carbonate fuel cell power plants. Energy, 83, 734-748, 2015.

14. Busse, M.R., Lacetera, N., Pope, D.G., Silva-Risso, J., \& Sydnor, J.R. Estimating the effect of salience in wholesale and retail car markets. American Economic Review, 103(3), 575-579, 2013.

15. Carvalho, A.N., Oliveira, F., \& Scavarda, L.F. Tactical capacity planning in a real-world ETO industry case: A robust optimization approach. International Journal of Production Economics, 180, 158-171, 2016.

16. Charnes, A., \& Cooper, W.W. Chance-constrained programming. Management Science, 6(1), 73-79, 1959.

17. Chen, X., Sim, M., \& Sun, P. A robust optimization perspective on stochastic programming. Operations Research, 55(6), 1058-1071, 2007.

18. Chen, X., Sim, M., Sun, P., \& Zhang, J. A linear decision-based approximation approach to stochastic programming. Operations Research, 56(2), 344-357, 2008.

19. Colson, B., Marcotte, P., \& Savard, G. Bilevel programming: A survey. 4OR, 3(2), 87-107, 2005

20. Dantzig, G.B. Linear programming under uncertainty. Management Science, 1(3-4), 197-206, 1955.

21. Dempe, S. Foundations of bilevel programming. Springer Science \& Business Media, 2002 .

22. Dempe, S., \& Zemkoho, A.B. On the Karush-Kuhn-Tucker reformulation of the bilevel optimization problem. Nonlinear Analysis: Theory, Methods \& Applications, 75(3), 12021218,2012

23. Dempe, S., \& Zemkoho, A.B. The bilevel programming problem: reformulations, constraint qualifications and optimality conditions. Mathematical Programming, 138(1-2), 447-473, 2013.

24. Fisher, M.L. What is the right supply chain for your product? Harvard Business Review (March-April), 105-116, 1997. 
25. Ellsberg, D. Risk, ambiguity and savage axioms. Quarterly Journal of Economics, 75(4), 643-669, 1961.

26. Gorissen, B.L., \& Den Hertog, D. Robust counterparts of inequalities containing sums of maxima of linear functions. European Journal of Operational Research, 227(1), 30-43, 2013.

27. Graves, S.C. Uncertainty and production planning. In Planning Production and Inventories in the Extended Enterprise (pp. 83-101), Springer US, 2011.

28. Guo, L., Lin, G.H., \& Ye, J.J. Solving mathematical programs with equilibrium constraints. Journal of Optimization Theory and Applications, 166 (1), 234-256, 2015.

29. Guo, P. One-shot decision approach and its application to duopoly market. International Journal of Information and Decision Sciences, 2(3), 213-232, 2010.

30. Guo, P., Yan, R., \& Wang, J. Duopoly market analysis within one-shot decision framework with asymmetric possibilistic information. International Journal of Computational Intelligence Systems, 3(6), 786-796, 2010.

31. Guo, P. One-shot decision theory. Systems, Man and Cybernetics, Part A: Systems and Humans, IEEE Transactions on, 41(5), 917-926, 2011.

32. Guo, P., \& Ma, X. Newsvendor models for innovative products with one-shot decision theory. European Journal of Operational Research, 239(2), 523-536, 2014.

33. Guo, P., \& Li, Y. Approaches to multistage one-shot decision making. European Journal of Operational Research, 236(2), 612-623, 2014.

34. Gyulai, D., Pfeiffer, A., \& Monostori, L. Robust production planning and control for multi-stage systems with flexible final assembly lines. International Journal of Production Research, 1-17, 2016

35. Hanasusanto, G.A., Roitch, V., Kuhn, D., \& Wiesemann, W. A distributionally robust perspective on uncertainty quantification and chance constrained programming. Mathematical Programming, 151(1), 35-62, 2015.

36. Higle, J.L., \& Kempf, K.G. Production planning under supply and demand uncertainty: A stochastic programming approach. In Stochastic Programming (pp. 297-315), Springer New York, 2011.

37. Ho, C.J. Evaluating the impact of operating environments on MRP system nervousness. International Journal of Production Research, 27(7), 1115-1135, 1989.

38. Hoheisel, T., Kanzow, C., \& Schwartz, A. Theoretical and numerical comparison of relaxation methods for mathematical programs with complementarity constraints. Mathematical Programming, 1-32, 2013.

39. Kahneman, D., \& Tversky, A. Prospect theory: An analysis of decision under risk. Econometrica, 47(2), 263-292, 1979.

40. Kazemi Zanjani, M., Nourelfath, M., \& Ait-Kadi, D. A multi-stage stochastic programming approach for production planning with uncertainty in the quality of raw materials and demand. International Journal of Production Research, 48(16), 4701-4723, 2010.

41. Koca, E., Yaman, H., \& Akturk, M.S. Stochastic lot-sizing with controllable processing times. Omega, 53, 1-10, 2015.

42. Lin, G.H., Xu, M., \& Ye, J.J. On solving simple bilevel programs with a nonconvex lower level program. Mathematical Programming, 144(1-2), 277-305, 2014.

43. Luo, Z.Q., Pang, J.S., \& Ralph, D. Mathematical programs with equilibrium constraints. Cambridge University Press, 1996.

44. Miller, B.L., \& Wagner, H.M. Chance constrained programming with joint constraints. Operations Research, 13(6), 930-945, 1965.

45. Mula, J., Poler, R., Garcia-Sabater, J.P., \& Lario, F.C. Models for production planning under uncertainty: A review. International Journal of Production Economics, 103(1), 271$285,2006$.

46. Nasiri, G.R., Zolfaghari, R., \& Davoudpour, H. An integrated supply chain production distribution planning with stochastic demands. Computers \& Industrial Engineering, 77, 35-45, 2014.

47. Nemirovski, A., \& Shapiro, A. Convex approximations of chance constrained programs. SIAM Journal on Optimization, 17(4), 969-996, 2006.

48. Orquin, J.L., \& Loose, S.M. Attention and choice: A review on eye movements in decision making. Acta Psychologica, 144(1), 190-206, 2013.

49. Outrata, J.V. On the numerical solution of a class of Stackelberg problems. Mathematical Methods of Operations Research, 34(4), 255-277, 1990. 
50. Pagnoncelli, B.K., Ahmed, S., \& Shapiro, A. Sample average approximation method for chance constrained programming: theory and applications. Journal of Optimization Theory and Applications, 142(2), 399-416, 2009.

51. Savage, L.J. The Foundations of Statistics. New York, NY: Wiley, 1954.

52. Scheel, H., \& Scholtes, S. Mathematical programs with complementarity constraints: Stationarity, optimality, and sensitivity. Mathematics of Operations Research, 25(1), 1$22,2000$.

53. Sen, A. The US fashion industry: a supply chain review. International Journal of Production Economics, 114(2), 571-593, 2008.

54. Shapiro, A., Dentcheva, D., \& Ruszczyński, A. Lectures on stochastic programming: modeling and theory. Society for Industrial and Applied Mathematics, 2009.

55. Shi, J., Zhang, G., \& Sha, J. Optimal production planning for a multi-product closed loop system with uncertain demand and return. Computers \& Operations Research, 38(3), 641-650, 2011.

56. Sodhi, M.S., \& Tang, C.S. Modeling supply-chain planning under demand uncertainty using stochastic programming: A survey motivated by asset-liability management. International Journal of Production Economics, 121(2), 728-738, 2009.

57. Starmer, C. Developments in non-expected utility theory: The hunt for a descriptive theory of choice under risk. Journal of Economic Literature, 38(2), 332-382, 2000.

58. Stephen, B., \& Vandenberghe, L. Convex optimization. Cambridge university press, 2004.

59. Stewart, N., Hermens, F., \& Matthews, W.J. Eye movements in risky choice. Journal of Behavioral Decision Making, 29(2-3), 116-136, 2016.

60. Tang, L., Che, P., \& Liu, J. A stochastic production planning problem with nonlinear cost. Computers \& Operations Research, 39(9), 1977-1987, 2012.

61. Thomassey, S. Sales forecasts in clothing industry: The key success factor of the supply chain management. International Journal of Production Economics, 128(2), 470-483, 2010

62. von Neumann, J., \& Morgenstern, O. Theory of games and economic behavior. Princeton, NJ: Princeton University Press, 1944.

63. Wang, C., \& Guo, P. Behavioral models for first-price sealed-bid auctions with the one-shot decision theory. European Journal of Operational Research, 261(3), 994-1000, 2017.

64. Wazed, M., Ahmed, S., \& Nukman, Y. A review of manufacture resources planning models under different uncertainties: state-of the-art and future directions. South Africa Journal of Industrial Engineering, 21(1), 17-33, 2010.

65. Wiesemann, W., Kuhn, D., \& Sim, M. Distributionally robust convex optimization. Operations Research, 62(6), 1358-1376, 2014.

66. Yanikoğlu, I., \& Kuhn, D. Decision rule bounds for two-stage stochastic bilevel programs. SIAM Journal on Optimization, 28(1), 198-222, 2018

67. Ye, J.J., \& Zhu D.L. Optimality conditions for bilevel programming problems. Optimization, 33(1), 9-27, 1995.

68. Zymler, S., Kuhn, D., \& Rustem, B. Distributionally robust joint chance constraints with second-order moment information. Mathematical Programming, 137(1-2), 167-198, 2013.

69. Zhu, X., \& Guo, P. The one-shot decision theory based production planning models. In Proceedings of IEEE International Conference on Industrial Engineering and Engineering Management (pp. 789-792), December 4-7, 2016.

70. Zhu, X., \& Guo, P. Approaches to four types of bilevel programming problems with nonconvex nonsmooth lower level programs and their applications to newsvendor problems. Mathematical Methods of Operations Research, 86(2), 255-275, (2017). 